\title{
Benchmarking Density Functionals for Chemical Bonds of Gold
}

\section{Kepp, Kasper Planeta}

Published in:

Journal of Physical Chemistry Part A: Molecules, Spectroscopy, Kinetics, Environment and General Theory

Link to article, DOI:

10.1021/acs.jpca.6b12086

Publication date:

2017

Document Version

Peer reviewed version

Link back to DTU Orbit

Citation (APA):

Kepp, K. P. (2017). Benchmarking Density Functionals for Chemical Bonds of Gold. Journal of Physical Chemistry Part A: Molecules, Spectroscopy, Kinetics, Environment and General Theory, 121(9), 2022-2034. https://doi.org/10.1021/acs.jpca.6b12086

\section{General rights}

Copyright and moral rights for the publications made accessible in the public portal are retained by the authors and/or other copyright owners and it is a condition of accessing publications that users recognise and abide by the legal requirements associated with these rights.

- Users may download and print one copy of any publication from the public portal for the purpose of private study or research.

- You may not further distribute the material or use it for any profit-making activity or commercial gain

- You may freely distribute the URL identifying the publication in the public portal

If you believe that this document breaches copyright please contact us providing details, and we will remove access to the work immediately and investigate your claim 


\section{Benchmarking Density Functionals for Chemical Bonds of Gold}

Kasper P. Kepp*

Technical University of Denmark, DTU Chemistry, Building 206, 2800 Kgs. Lyngby, DK - Denmark.

* Corresponding Author. Phone: +045 452524 09. E-mail: kpj@kemi.dtu.dk 


\section{Abstract.}

Gold plays a major role in nanochemistry, catalysis, and electrochemistry. Accordingly, hundreds of studies apply density functionals to study chemical bonding with gold, yet there is no systematic attempt to assess the accuracy of these methods applied to gold. This paper reports a benchmark against 51 experimental bond enthalpies of $\mathrm{AuX}$ systems and seven additional polyatomic and cationic molecules. 12 density functionals were tested, covering meta functionals, hybrids with variable $\mathrm{HF}$ exchange, double-hybrid, dispersion-corrected and non-hybrid GGA functionals. The defined benchmark data set probes all types of bonding to gold from very electronegative halides that force $\mathrm{Au}^{+}$ electronic structure, via covalently bonded systems, hard and soft Lewis acids and bases that either work against or complement the softness of gold, the $\mathrm{Au}_{2}$ molecule probing gold's bond with itself, and weak bonds between gold and noble gases. Zero-point vibrational corrections are relatively small for $\mathrm{Au}-\mathrm{X}$ bonds, $\sim 11-12 \mathrm{~kJ} / \mathrm{mol}$ except for $\mathrm{Au}-\mathrm{H}$ bonds. Dispersion typically provides $\sim 5 \mathrm{~kJ} / \mathrm{mol}$ of the total bond enthalpy but grows with system size and is $10 \mathrm{~kJ} / \mathrm{mol}$ for AuXe and AuKr. HF exchange and LYP correlation produce weaker bonds to gold. Most functionals provide similar trend accuracy, though somewhat lower for M06 and M06L, but very different numerical accuracy. Notably, PBE and TPSS functionals with dispersion display the smallest numerical errors and very small mean signed errors $(0-6 \mathrm{~kJ} / \mathrm{mol})$, i.e. no bias towards over- or under-binding. Errors are evenly distributed vs. atomic number, suggesting that relativistic effects are treated fairly; the mean absolute error is almost halved from B3LYP (45 kJ/mol) to TPSS and PBE (23 kJ/mol, including difficult cases); $23 \mathrm{~kJ} / \mathrm{mol}$ is quite respectable considering the diverse bonds to gold and the complication of relativistic effects. Thus, studies that use DFT with effective core potentials for gold chemistry, with no alternative due to computational cost, are on solid ground using TPSS-D3 or PBE-D3. 


\section{Introduction.}

The chemistry of gold is very rich and highly specialized considering the position of gold in the activity series, due to a combination of unique chemical properties relating to its polarizability and relativistic effects $^{1,2,3}$, and because gold is one of the least oxophilic elements that exist ${ }^{4}$. Accordingly, gold serves an enormously broad range of purposes across chemistry: Gold surfaces are vital in many types of nanoscience $^{5}$ and heterogeneous catalysis ${ }^{6}$ and as frameworks for other processes such as molecular magnets $^{7}$. The unique chemical properties of gold clusters ${ }^{8}$ make them suitable as catalytic facilitators $^{9,10}$ or even as direct catalysts of a variety of reactions ${ }^{11}$. The contra-intuitive ability of gold cations to associate in stable interactions with hydrogen-bond like interaction energies (aurophilic interactions) are to a large degree due to the substantial polarizability of gold ${ }^{12}$.

Because of the many uses of gold, there is a large need to understand and predict chemical interactions with gold ${ }^{6,8}$. To achieve this, accurate theoretical computations are necessary that can reproduce, explain, and predict observed data. Currently, density functional theory (DFT) is the only viable method for studying gold chemistry in general except for very small systems such as small clusters: When studying catalysis, materials, and surfaces, DFT is the only option due to the scaling of cpu requirements with system size $\mathrm{e}^{13,14,15}$. Thus, DFT has been used in hundreds of studies to understand gold molecular systems ${ }^{2}$. Given the scarcity of experimental benchmark data, accuracy is generally based on benchmarking calculations against a few equilibrium structures or binding energies, often using $a b$ initio methods in the absence of experimental data ${ }^{16,17,18}$. These standard approaches almost exclusively use effective core potentials, while explicit treatment of spin-orbit coupling is beyond computational reach for larger gold-containing systems ${ }^{2,19}$. Mostly, the choice of functional is

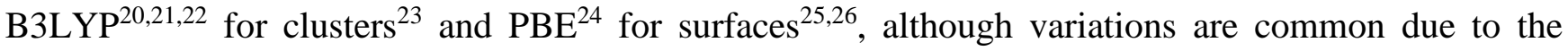
different preferences of research groups ${ }^{2}$. Finally, since standard dispersion corrections only became widely available the last five years, dispersion corrections are not generally included in these studies. 
Notable previous work studied the performance of several density functionals against CCSD(T) for $\mathrm{Cu}, \mathrm{Ag}$, and $\mathrm{Au}-$ systems different from this work $^{27}$, revealing important effects on the binding interactions of coin metals to ligands. Also, a detailed benchmark of M06, TPSS, B3LYP, and BP86 against $\operatorname{CCSD}(\mathrm{T})$ calculations for gold-carbon adducts has been reported ${ }^{28}$. A study of $\mathrm{AuBe}, \mathrm{AuMg}$, AuCa, AuSr, and AuBa has been carried out using CCSD(T) and TPSSh ${ }^{29}$. However neither of these studies provided a systematic and direct benchmark to experimental thermochemical, which seems desirable in order to assess the performance of DFT for gold chemistry more generally.

It is therefore of substantial importance to assess the accuracy of these standard approaches more systematically against available experimental thermochemical data. The acronym "DFT" covers a very diverse range of functionals with different philosophies of design, parameterization, mathematical form, and approximations, and various extent of self-interaction error ${ }^{13}$. It is relevant to ask how accurate these approaches really are, and whether the literature conclusions based on such energy calculations can be justified. The most notable errors that will potentially make such calculations inaccurate and their interpretations of gold chemistry invalid are i) the differences in the functional itself, such as how much Hartree-Fock exchange it includes, which changes bond energies of metalligand bonds by many tens of $\mathrm{kJ} / \mathrm{mol}^{30}$ ii) the suitability of the effective core potentials for modeling the relativistic effects that are instrumental to gold chemistry ${ }^{1}$; iii) the inclusion of thermal effects such as zero-point energies of the gold-ligand or gold-gold vibrations which change bond enthalpies substantially ${ }^{30}$, and iv) the importance of dispersion, which is important in understanding gold's chemistry due to the polarizable nature of the gold atom ${ }^{219}$. These various effects are systematic and will change the computed thermochemistry substantially ${ }^{13}$. In this light, a systematic benchmarking of these standard DFT approaches to the study of chemical bonds involving gold seems warranted. 


\section{Methods.}

Experimental Data. Experimental bond dissociation enthalpies (BDH) at standard elevated temperature conditions, 1 atm gas phase, $298.15 \mathrm{~K}$ were collected from the 2014-updated version of the CRC Handbook of Chemistry and Physics ${ }^{31}$. The full list of $51 \mathrm{BDH}$ values for Au-X bonds can be found in Supporting Information, Table S1. All experimental data found were used without exclusion of any data in the benchmark. Additionally, experimental $\mathrm{BDH}$ values for $\mathrm{AuNH}_{3}, \mathrm{AuCH}_{3}, \mathrm{AuC}_{6} \mathrm{H}_{6}$, and the cations $\mathrm{AuCO}^{+}, \mathrm{AuH}^{+}, \mathrm{AuNH}_{3}{ }^{+}$, and $\mathrm{AuXe}^{+}$were included in the benchmark (Supporting Information, Table S2). The value for AuLa is cited as $457 \mathrm{~kJ} / \mathrm{mol}$ in the CRC Handbook. However, this value stands out in the experimental data series, and a Reviewer pointed out that a more reasonable value of $335 \mathrm{~kJ} / \mathrm{mol}$ was determined by Gingerich and Finkbeiner ${ }^{32}$; thus the latter value, which is within the expected range of experimental data, was used in this work.

Geometry Optimizations. All computations were performed using the Turbomole software, version $7.0^{33}$. Both the neutral atoms of and the molecules were optimized in all possible spin states to ensure that the lowest ground state could be identified, starting from high-spin states first to ensure convergence to the lowest open-shell low-spin states where applicable. The resolution of identify approximation $^{34}$ was used to speed up calculations throughout this work. The molecules were all optimized using two different functionals and including dispersion, PBE-D3 ${ }^{2435}$ and TPSSh-D3 ${ }^{3637}$, to account for geometric effects of functional. AuX bonds were similar within $\sim 0.03 \AA$ except for a few extreme cases with very long weak bonds such as AuKr (Supporting Information, Table S3) and gave similar subsequent single-point energies when computed with the same functional within less than 1 $\mathrm{kJ} / \mathrm{mol}$ in all cases except five: AuU $(1.3 \mathrm{~kJ} / \mathrm{mol}$ difference $)$, AuXe $(1.2 \mathrm{~kJ} / \mathrm{mol})$, AuNd $(3.8 \mathrm{~kJ} / \mathrm{mol})$, AuCe $(5.7 \mathrm{~kJ} / \mathrm{mol})$, and $\mathrm{AuPr}(11.2 \mathrm{~kJ} / \mathrm{mol})$. Because of this, geometry differences for the functionals did not affect final energies and essentially all of the difference in computed gold thermochemistry thus 
lies in the subsequent single-point energies. The total list of computed electronic energies of optimized electronic states of the Au-X molecules is given in Supporting Information, Table S4.

The main purpose of this work is to test the performance of the standard methodology applied in the literature, which generally relies on triple-zeta type basis sets with effective core potentials as the approximation for relativistic effects, without any explicit account of e.g. spin-orbit coupling. To mimic such standard approaches the def2-TZVP basis sets and ecp2 effective core potentials from the Turbomole package ${ }^{38}$ were used for both geometry optimization and subsequent energy calculation. These basis sets have been systematically optimized for all elements studied; therefore all elements are treated in the same comparative basis set regime, so that differences in basis function types and parameters are not causing differences in performance between molecules. This is an important requirement achieved specifically with the universal def2 basis sets. The basis set is large enough that basis set effects are expected to only a few $\mathrm{kJ} / \mathrm{mol}$ based on previous studies comparing quadruple zeta polarized basis sets to these types of TZVP basis sets also for M-L bond strengths ${ }^{39}$.

Functionals Studied. Subsequent to geometry optimization, the single point energies of the deduced ground states were computed on the PBE-D3 optimized ground state geometries using the following different functionals: PBE-D3 ${ }^{24}$, PBE0-D3 ${ }^{40}$, TPSSh-D3 and TPSS-D3 ${ }^{3637}$, B3LYP ${ }^{202141}$ and B3LYP-D3 ${ }^{20,21}$, BLYP ${ }^{2142}$, B3LYP*43,44 with $15 \%$ HF exchange, B3LYP10 with $10 \%$ HF exchange (customized for this study using Turbomole's built-in feature enabling this option), M06 ${ }^{45}$, M06L ${ }^{46}$, and B2PLYP ${ }^{47}$, with electronic energies converged to $10^{-7}$ a.u. Dispersion effects were estimated using the D3 correction $^{35}$. The MP2 energies for B2PLYP were also computed using the RI approximation ${ }^{48}$. The electronic single-point energies for the all $\mathrm{AuX}$ molecules and $\mathrm{X}$ atoms can be found in Supporting Information, Tables S5 and S6, respectively. Bond dissociation enthalpies were computed from the energy of the AuX system, minus the energies of the Au atom and the $\mathrm{X}$ fragment (either an atom or a molecule), and adding thermal vibrational corrections for the bond enthalpy as specified below. 
Zero-Point Vibrational Energy and Thermal Enthalpy Estimates. The frequencies of all optimized ground states were computed at the same level as geometry optimization, i.e. def2-TZVP. The zero point energies (ZPEs) were subtracted from the energies of each molecular species involved in the benchmark bond dissociation enthalpy. The enthalpy corrections were obtained from thermodynamic calculations at $298 \mathrm{~K}$ using the state function from the frequencies scaled by a scale factor of 0.99 (the exact choice of this scale factor between 0.95 and 1 has no effect on the final computed energies due to the magnitude of the ZPE, as seen in Supporting Information, Table S7 where these data are compiled. These calculations were done at $298.15 \mathrm{~K}$ to compare to the experimental temperature.

Coupled-Cluster Computations. Methods such as coupled-cluster are not generally applicable to gold chemistry due to the size of gold clusters, surfaces, and catalytic systems, and this method is very basis set sensitive and even then still approximate in its treatment of relativistic effects ${ }^{29}$, which is why this paper uses direct experimental data rather than $\operatorname{CCSD}(\mathrm{T})$ data as reference data; still $\operatorname{CCSD}(\mathrm{T})$ comparison to DFT at same basis level provides a useful comparison of method behavior.

Such calculations were carried out using the resolution of identify approximation as implemented in Turbomole ${ }^{49}$ for all the atoms, ions and molecules to compute the BDH, using tighter convergence criteria on both density and energy $\left(10^{-7}\right.$ a.u. for both). The HF reference configurations are critically important in providing the correct $\operatorname{CCSD}(\mathrm{T})$ energy and were thus carefully converged, often upon multiple attempts from higher lying spin states, to identify the lowest-energy HF reference configuration; this procedure is non-trivial as many of the atoms and some molecules have close-lying electronic configurations and easily converge to metastable configurations. However, the final BDHs computed serve as a sanity check of these configurations, and show that the appropriate configurations have indeed been identified. The D1 diagnostics were also computed and the electronic CCSD(T) energies for all systems are given together with the DFT results in the Supporting information. 


\section{Results and Discussion.}

Bond Dissociation Enthalpies of AuX Systems. The experimental data set of BDH values for $51 \mathrm{AuX}$ molecules (Supporting Information, Table S1) covers most of the periodic table, with elements representing all periods and both s-, p-, d- and f-block elements. The experimental data are shown in Figure $1 \mathrm{~A}$ vs. atomic number. The benchmark data set probes all types of bonding to gold from very electronegative halides that force $\mathrm{Au}^{+}$electronic structure, via covalently bonded systems with similar electronegativity as gold, small hard Lewis acids and large Lewis acids and bases that either work against or complement the substantial polarizability and softness of gold, the $\mathrm{Au}_{2}$ molecule probing gold's bond with itself, and very weak bonds between gold and noble gases of Kr and Xe that stick out near zero in Figure 1A. The noble gas gold adducts are known to have very weak but favorable binding that is roughly half due to relativistic effects and half due to dispersion ${ }^{50}$. The fact that all these systems are studied with the exact same methodology and basis set types makes it possible to compare the bonding types to gold more generally than previously done.

The first feature that can be separated out from these bond enthalpies is the dispersion contribution to the $\mathrm{Au}-\mathrm{X}$ bonds. This contribution cannot be measured experimentally but can be computed from the difference between the DFT results without and with dispersion correction. Figure 1B shows the values obtained from B3LYP and B3LYP-D3, as can be found numerically in Supporting Information, Table S6. Although each functional has its own D3 correction, these dispersion corrections are numerically similar and of the order of $\sim 5 \mathrm{~kJ} / \mathrm{mol}$ except for the noble gas adducts where they contribute $10 \mathrm{~kJ} / \mathrm{mol}$ and dominate the total bond enthalpy. The DFT methods would not predict any bonding between gold and the noble gases without dispersion included, illustrating the importance of dispersion corrections to DFT in such cases. For larger molecules, the importance of 
dispersion generally increases, as seen from the trend line, but already for these diatomic molecules it is a systematic factor, although still numerically modest.
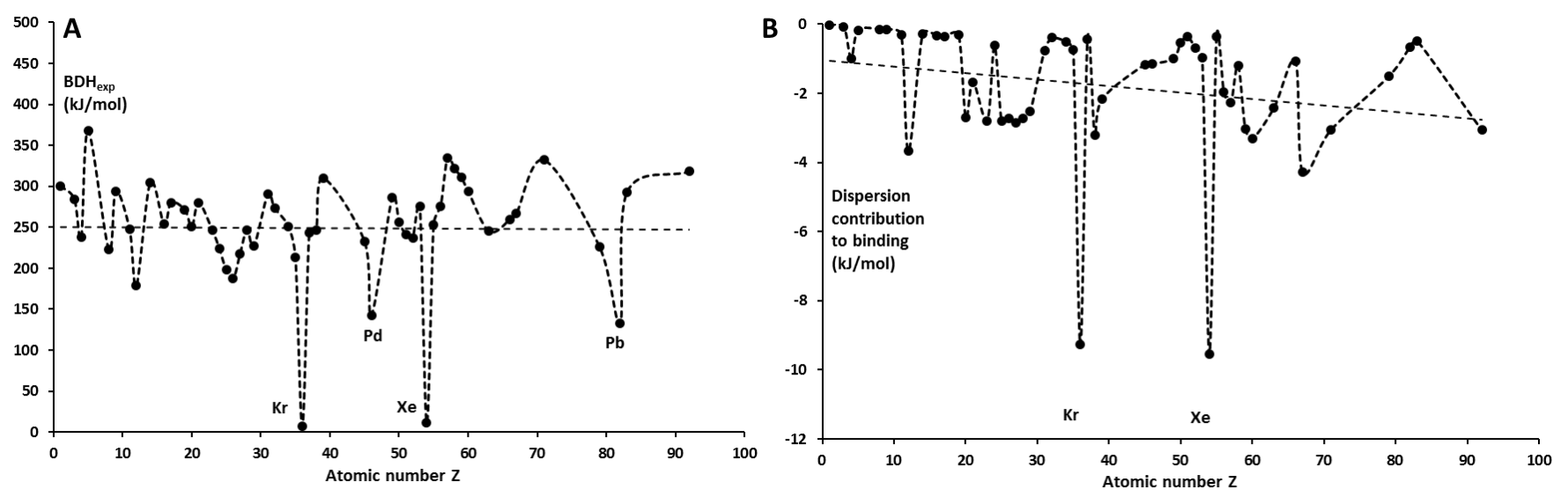

Figure 1. Bonds to gold: A) Experimental bond dissociation enthalpies (in $\mathrm{kJ} / \mathrm{mol}$ ) of AuX molecules vs. atomic number; B) Computed contribution of dispersion forces to the bonding, in $\mathrm{kJ} / \mathrm{mol}$ (estimated from B3LYP-D3/def2-TZVP).

Performance of DFT Applied to Gold Bonding. Figure 2 shows the experimental BDHs plotted against the computed BDHs for the 12 different functionals and $\operatorname{CCSD}(\mathrm{T})$. The total thermal vibrational corrections to these values are fairly constant around 11-12 kJ/mol for the AuX diatomic molecules with the exception of AuH which, due to the small reduced mass of this molecule, has a larger correction of $22 \mathrm{~kJ} / \mathrm{mol}$ (Table S7). These corrections are included in all BDH estimates shown in Figure 2; the numerical final computed BDH values can be found in Supporting Information, Table S8 and Table S9. AuKr and AuXe were left out in Figure 2 as the two small values of render the correlation much larger $\left(\mathrm{R}^{2} \sim 0.76-0.78\right)$ and gives undue weight to these extreme points. Figure 2 without them more clearly shows the substantial spread obtained using different functionals. 


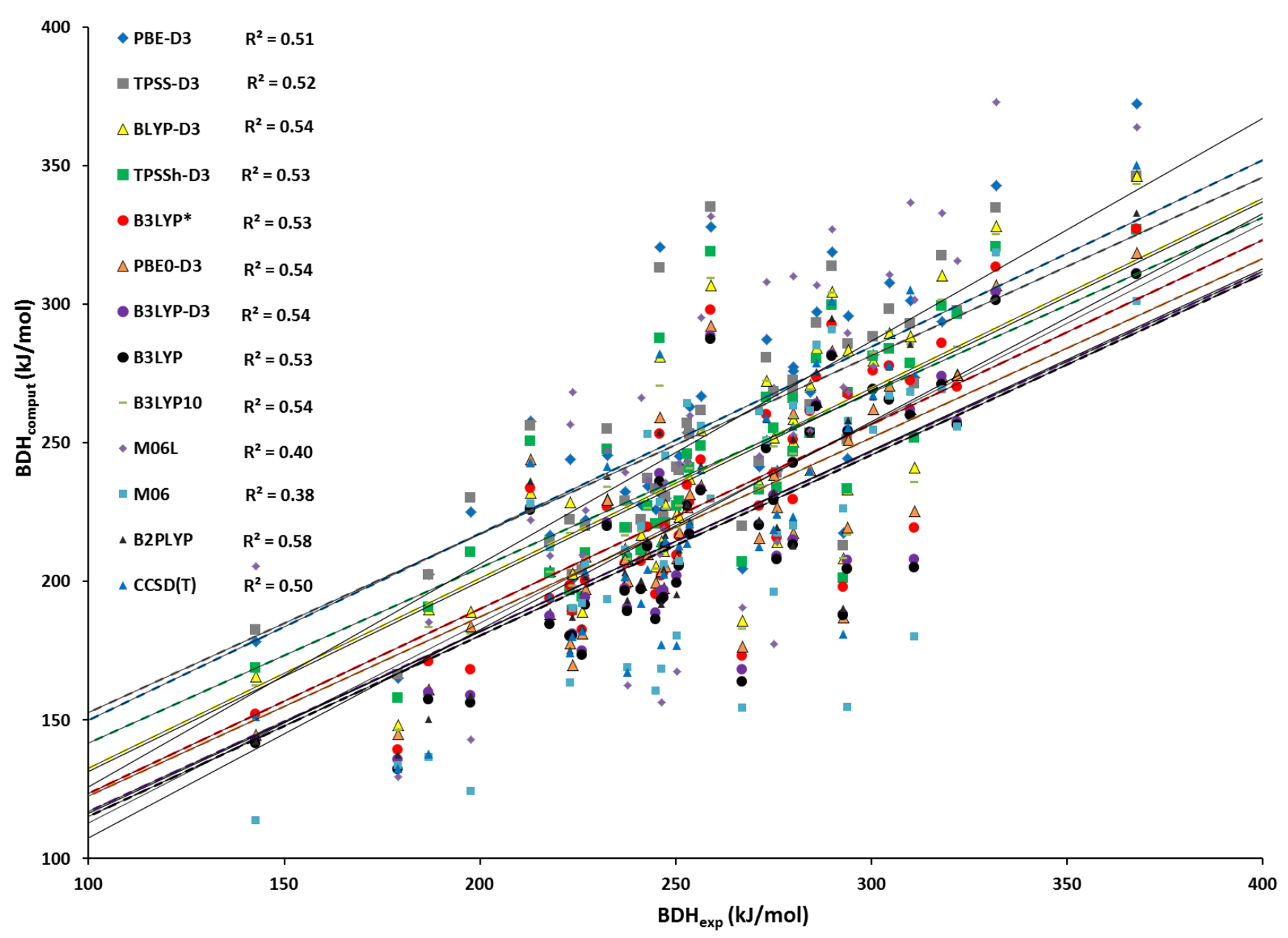

Figure 2. Experimental vs. computed bond dissociation enthalpies (in $\mathrm{kJ} / \mathrm{mol}$ ) of gold-containing diatomic molecules (expect the noble gas adducts AuXe and AuKr).

It can be seen from Figure 2 that despite this spread, all the functionals perform very similar in terms of their general trend correlation for BDHs, which can be explained as due to the fact that DFT generally is very good at providing differential bonding information in comparisons, where systematic errors tend to cancel. The functionals display $\mathrm{R}^{2}$ values in the range $0.38-0.58$; however without M06 and M06L, the range is only $0.50-0.58$. Thus, in terms of ability to reproduce trends in bond strengths, all methods except M06 and M06L perform equally well. The poor performance of M06 and M06L is curious but may be related to the sacrifice of fundamental trend accuracy in heavily parameterized 
functionals outside their parameterization range; if so, this mirrors a recent report ${ }^{51}$ that these two functionals are potentially less "universal" than other functionals studied here. It is also notable that $\operatorname{CCSD}(\mathrm{T})$ has a trend prediction that is similar to most functionals, and that B2PLYP stands out with a somewhat better trend prediction than any other method.

Figure 3 shows the signed errors for all studied methods (numerical data can be found in Supporting Information, Table S10). It is notable that the majority of the studied functionals are hybrid functionals that tend to underestimate chemical metal-ligand bond strengths (i.e. under-bind) as shown previously in benchmarks across all three rows of the d-block ${ }^{30,39}$. This phenomenon is equally true for all three rows of the d-block i.e. relativistic effects do not change this preference, suggesting that the effective core potentials work well at least in a comparative sense across the three periods ${ }^{30}$.

Table 1 shows the mean signed errors and mean absolute errors for all functionals studied. Specifically, the hybrids B3LYP, B3LYP-D3, B3LYP*, PBE0-D3 and TPSSh-D3 all underestimate $\mathrm{Au}-\mathrm{X}$ bond strengths on average by $39,38,29,33$, and $17 \mathrm{~kJ} / \mathrm{mol}$, the least for TPSSh which only includes $10 \%$ HF exchange. Calculation with a B3LYP version including only $10 \%$ HF exchange (B3LYP10) shows that this is indeed due to $10 \%$ HF exchange, giving very similar performance as TPSSh (19 vs. $17 \mathrm{~kJ} / \mathrm{mol}$ average underestimation of BDH). These findings are consistent with the previous findings that HF exchange weakens metal-ligand bonds ${ }^{39}$; this has also been observed in previous studies of gold chemistry ${ }^{28}$. Also BLYP under-binds by $17 \mathrm{~kJ} / \mathrm{mol}$ on average. Compared to the other non-hybrid GGA functional, PBE, this is consistent with the previous finding that the LYP correlation functional under-binds compared to other comparable correlation functionals ${ }^{39,52}$. From this analysis, one can conclude that the chemical bonds to gold follow the general bonding tendencies of the investigated functionals and reflect clearly the weaker-binding effect of HF exchange and LYP correlation. These two features add up in the B3LYP functional, making it the most under-binding of 
all studied methods together with M06 with 27\% HF exchange. Thus, most studied methods underbind, as evident from Figure 3.

In contrast, as seen from Figure 3, PBE-D3 and TPSS-D3 perform surprisingly accurately with signed errors that are almost zero. As summarized in Table 1, they produce MSEs of -2 and $-4 \mathrm{~kJ} / \mathrm{mol}$ for this large and diverse data set that covers most of the periodic table, which is a very positive finding. Previous studies of some saturated carbon adducts with gold reached a similar conclusion that TPSS performs well ${ }^{28}$. Also, the local meta functional M06L has a low signed error $(-5 \mathrm{~kJ} / \mathrm{mol})$. These errors show that these three methods do not have a bias towards under- or overbinding.

Equally important, the corresponding mean absolute errors (MAE) are listed in Table 1 (full list of absolute errors can be found in the Supporting Information, Table S11). The MAE generally follows the trend for MSE because the trend predictions $\left(\mathrm{R}^{2}\right)$ are similar, except for M06L which had a small MSE but an average MAE of $32 \mathrm{~kJ} / \mathrm{mol}$. In terms of absolute errors, a MAE of $23 \mathrm{~kJ} / \mathrm{mol}$ is seen for PBE-D3 and TPSS-D3, whereas the highest for M06 and B3LYP (44/45 kJ/mol). Thus, MAEs of TPSS and PBE are reduced to half that of the commonly used B3LYP functional. After PBE and TPSS, the non-hybrid GGA BLYP, and TPSSh and B3LYP10 both with 10\% HF exchange have the smallest MAE. Again, TPSSh and B3LYP10 perform very similar showing that HF exchange dominates the differences in computed bond strengths, a finding that is further confirmed by the order of performance BLYP > B3LYP10 > B3LYP* > B3LYP $(0,10,15$, and 20\% HF exchange). The smallest root mean square deviations from experimental values (RMSD) are also obtained with TPSS and PBE (32 kJ/mol) but closely followed by TPSSh, B3LYP10, and BLYP. Thus, combing the numerical accuracy (MAE, RMSD), the bonding bias (MSE) and the trend prediction accuracy $\left(\mathrm{R}^{2}\right)$ in total, PBE-D3 or TPSS-D3 clearly perform most accurately in this benchmark using a model approach that is likely to be routinely accessible for gold chemistry. 
Table 1. Mean Signed (MSE) and Mean Absolute (MAE) Errors, Root-Mean-Square Deviation (RMSD), and Squared Correlation Coefficients $\left(\mathbf{R}^{2}\right)$ of Tested Methods (in kJ/mol).

\begin{tabular}{|l|l|l|l|l|l|l|l|}
\hline Method & B3LYP-D3 & B3LYP* & BLYP-D3 & PBE0-D3 & PBE-D3 & TPSSh-D3 & TPSS-D3 \\
\hline MSE & -38 & -29 & -17 & -33 & -2 & -17 & -4 \\
\hline MAE & 43 & 36 & 27 & 40 & 23 & 29 & 23 \\
\hline RMSD & 49 & 43 & 35 & 45 & 32 & 35 & 32 \\
\hline R $^{2}$ & 0.54 & 0.53 & 0.54 & 0.54 & 0.51 & 0.53 & 0.52 \\
\hline Method & B3LYP & B3LYP10 & M06L & M06 & B2PLYP & CCSD(T) & \\
\hline MSE & -39 & -19 & -5 & -38 & -29 & -27 & \\
\hline MAE & 45 & 29 & 32 & 44 & 35 & 41 & \\
\hline RMSD & 50 & 36 & 44 & 55 & 41 & 49 & \\
\hline R & 0.53 & 0.54 & 0.40 & 0.38 & 0.58 & 0.50 & \\
\hline
\end{tabular}




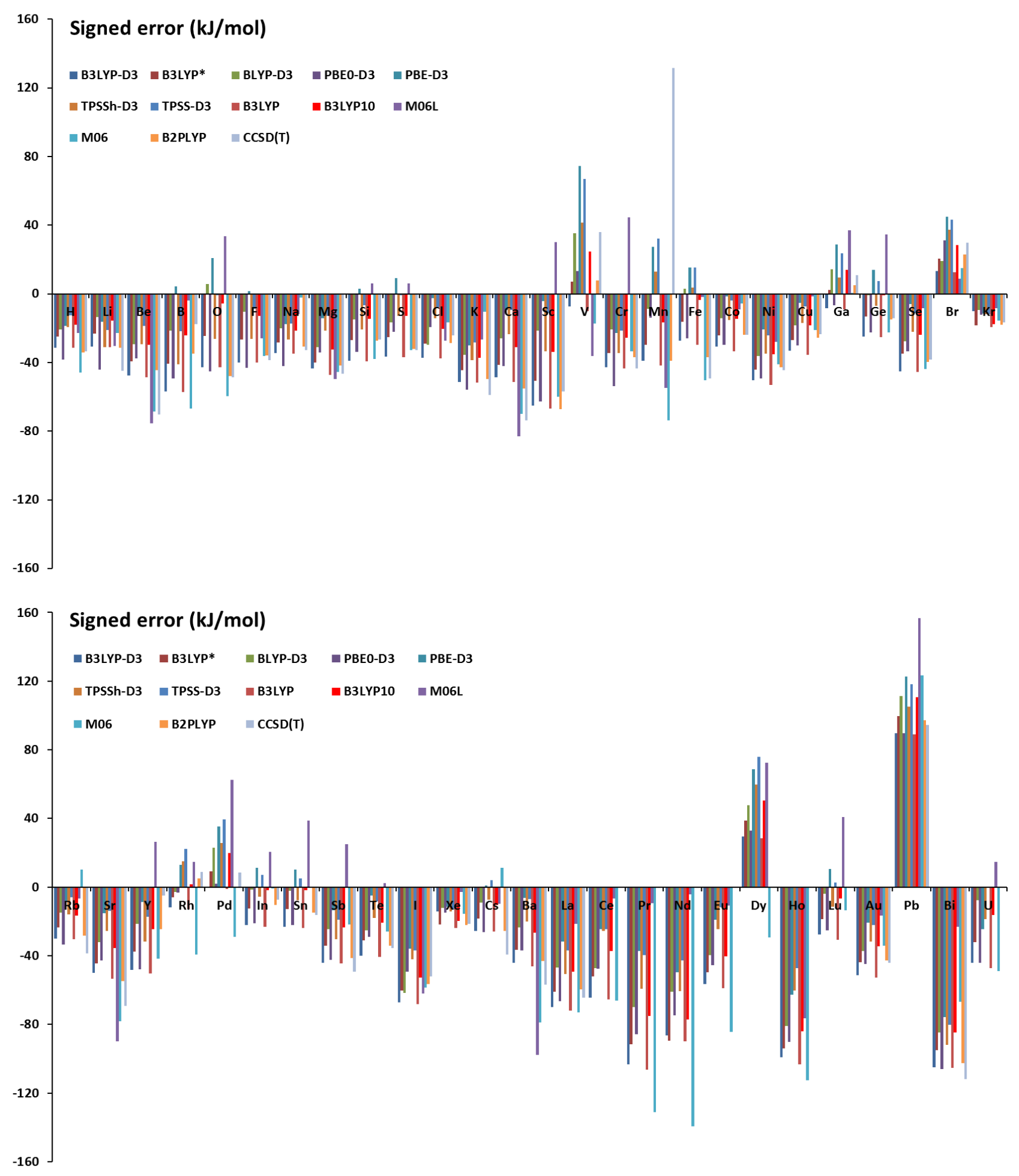

Figure 3. Signed errors in computed bond dissociation enthalpies $(\mathrm{kJ} / \mathrm{mol})$ for all AuX molecules. 
CCSD(T) Computations. To give an idea of the performance of post-Hartree-Fock correlated methods, all systems were computed also with CCSD(T)/def2-TZVP using tightly converged $\left(10^{-7}\right.$ a.u. in density and energy) HF reference configurations. These particular systems feature static and openshell correlation in particular for the atomic states required to compute the $\mathrm{BDH}$, and $\mathrm{CCSD}(\mathrm{T})$ cannot be applied to chemically relevant gold systems in catalysis and nonoscience. However, in previous benchmarks $\operatorname{CCSD}(\mathrm{T})$ has been used as the reference rather than experimental data. ${ }^{27,28}$ For comparison with DFT, the def2-TZVP basis set was used but a considerable basis set error is expected for $\mathrm{CCSD}(\mathrm{T})$ not present in the DFT calculations ${ }^{39}$. Previous work on $\mathrm{AuBe}, \mathrm{AuMg}, \mathrm{AuCa}, \mathrm{AuSr}, \mathrm{AuBa}{ }^{29}$ suggested that this leads to under-binding, so the comparison here is not a test of $\operatorname{CCSD}(\mathrm{T})$ but a comparison of correlation energy recovery within the same polarized triple-zeta basis set regime. The computed BDHs agree within $20 \mathrm{~kJ} / \mathrm{mol}$ with the corresponding CCSD(T)/def2-TZVPP values in previous work for these five systems (note that for these systems, def2-TZVPP and def2-TZVP are identical because the additional polarization function resides only on hydrogens $)^{29}$.

Figure 2 showed a trend prediction for $\operatorname{CCSD}(\mathrm{T})$ that resembled that of most density functionals. The errors in Table 1 show that overall, $\operatorname{CCSD}(\mathrm{T})$ performs similar to the high-HF fraction hybrids B3LYP (20\%) and PBE0 (25\%) for these systems; this indicates that CCSD(T) as a post-HF method still resembles somewhat the single-reference HF reference state. The D1 diagnostic for most of these molecules is generally larger than 0.05 , commonly larger than 0.1 and in several cases larger than 0.2 (see Figure S1, Supporting Information). Although accuracy can clearly be improved with larger basis sets and explicit spin-orbit coupling, $\operatorname{CCSD}(\mathrm{T})$ is not currently useful for studies of gold clusters and gold surfaces. The performance of TPSS and PBE is encouraging in this context. In the following, the $\operatorname{CCSD}(\mathrm{T})$ data are discussed in comparison to the density functionals where appropriate.

System-Specific Performance of DFT. The errors can be usefully divided into parts of the periodic table to determine if specific electronic configurations, shell structure, or heavy-atom effects 
change the relative accuracy of the methods. Figure 3 shows some outlier behavior in $\mathrm{AuPb}, \mathrm{AuV}$ and AuBr and AuDy which tend to have weaker bonds that what DFT predicts. The lanthanides tend to have slightly larger errors than average, but not in a substantial way. Generally, the most important observation is that errors are evenly distributed as a function of the atomic mass of $\mathrm{X}$, which means that the heavy atom effects do not build up systematic errors in the applied methodology. The average signed error of all methods is $-23 \mathrm{~kJ} / \mathrm{mol}$; for the first and second period this MSE is $-27 \mathrm{~kJ} / \mathrm{mol}$, very similar to the total average. For the third and fourth periods it is -20 and $-17 \mathrm{~kJ} / \mathrm{mol}$, again close to the total average. For the fifth period $(\mathrm{Rb}$ to $\mathrm{Xe})$ it is the smallest, $14 \mathrm{~kJ} / \mathrm{mol}$. For the remaining heavy atoms the MSE is $-29 \mathrm{~kJ} / \mathrm{mol}$, within the uncertainty expected from the errors. Thus, the heavy atom effect on errors is $\sim 10 \mathrm{~kJ} / \mathrm{mol}$, i.e. the effective core potentials used in this work accurately mimics heavy atom effects relative to light atoms, so that the treatment of the systems is uniformly accurate and not impaired by deficiencies in the description of relativistic effects. In this light, too negative assumptions have probably been made on the applicability of DFT to gold chemistry assuming that relativistic effects would not be well described; the present data are very encouraging but also mimic positive conclusions from DFT with effective core potentials reported previously for specific systems ${ }^{53}$.

Secondly, one can look at the errors divided into s-blocks, p-blocks, d-blocks, and f-blocks, to estimate systematic errors specific to electronic configurations such as promotion energies and the effect of inert pairs, which tend to be stabilized by relativistic effects. Remarkably, these errors are essentially uniformly distributed (i.e. not significantly different) as partly evident from Figure 3. For example, the computed MSE for the alkali metal gold adducts (AuLi, AuNa, AuK, AuRb, and AuCs) is $-25 \mathrm{~kJ} / \mathrm{mol}$ and MSE for the alkaline earth adducts (AuBe, AuMg, AuCa, AuSr, and AuBa) is -26 $\mathrm{kJ} / \mathrm{mol}$, both very close to the average $\operatorname{MSE}(-21 \mathrm{~kJ} / \mathrm{mol})$ of the full data set. For the gold-halide adducts (AuF, $\mathrm{AuCl}, \mathrm{AuBr}$, and $\mathrm{AuI})$ the $\mathrm{MSE}$ is $-18 \mathrm{~kJ} / \mathrm{mol}$ confirming this observation. In conclusion, the standard DFT methodology benchmarked here, is remarkable robust to electronic 
configuration effects, bonding type, shell structure, and relativistic effects beyond the effective core, and can achieve, for the PBE-D3 and TPSS-D3 methods, accuracy that is well competing with general computational chemistry. Of course this depends on the ability to converge the correct electronic configurations of atoms and molecules, which should be carefully quality controlled as this is not always trivial (the converged electronic energies of all involves atoms and molecules can be found in Supporting Information Tables S4 and S5).

Electronic States Computed by PBE. Because of the general good agreement with experimental BDH values, the PBE-computed ground state spin multiplicities are likely to be accurate estimates of the true spin multiplicity. Because of this relationship, this work provides also the electronic spin multiplicity of a large range of $\mathrm{AuX}$ molecules whose electronic ground states have not generally been characterized experimentally. The ground states are marked in bold in Supporting information, Table S4; most ground states are in the lowest spin quantum number, but there are many exceptions notably in the d- and f-block transition metal gold adducts such as $\mathrm{AuCr}, \mathrm{AuEu}, \mathrm{AuV}$, AuCo, AuDy, and AuRh, but also two p-block adducts, AuSb and AuBi, have higher multiplicity ground states according to the computations; similarly the equilibrium bond lengths are not generally known for these species; these predictions may be tested by experiments in the future.

Gold Bond-Strength Bond-Length Correlations. Above it was shown that some DFT methodologies, notably PBE-D3 and TPSS-D3 with the applied ZPE corrections and effective core potentials, reproduce experimental $\mathrm{Au}-\mathrm{X} \mathrm{BDHs}$ with MAEs of $\sim 23 \mathrm{~kJ} / \mathrm{mol}$, similar to typical computational chemistry, and with fairly uniform accuracy across the periodic table even with the data set covering a diverse range of bonds.

Figure 4 shows the relationship between optimized equilibrium bond lengths (in pm) and the computed BDH (using PBE-D3 as example) for all p-block elements (Figure 4A, $\mathrm{R}^{2}=0.76$ ) and for the 
alkali metal adducts (Figure $4 \mathrm{~B}, \mathrm{R}^{2}=0.64$ ), representing similar bonding patterns within each group but different from each other (i.e. ionic $\mathrm{Au}^{+}$for the p-block adducts except noble gasses and $\mathrm{Au}^{-}$for the s-block adducts). The relationship is a linear anti-correlation as generally expected. In the d-block, the bond lengths are very similar because all the metals binding to gold have very similar radii but their BDHs differ substantially due to the different electronic configurations of the adducts, notably with many high-spin configurations of the middle d-block. Accordingly, the relationship is weak $\left(\mathrm{R}^{2}=0.16\right.$, and in fact slightly reverse compared to normal bond strength bond length correlations, which is however not significant considering the $20-\mathrm{kJ} / \mathrm{mol}$ error bar in the $\mathrm{BDH}$ values). Corresponding data for all molecules (Supporting Information, Figure S2 A) show modest correlation with $\mathrm{R}^{2} \sim 0.27$ due to the many different types of bonding involved, including covalent bonding (e.g. AuSb and AuSi, see below), weak van der Waals interactions (AuXe, AuKr), ionic bonding where Au becomes cation (AuCl, AuF e.g.) or anion (AuK, AuLi e.g.). The correlation is increased if one considers only local parts of the periodic table, such as the light elements bonding with gold (Supporting Information Figure $\mathrm{S} 2 \mathrm{~B}, \mathrm{R}^{2}=0.46$ ) or the lanthanide-gold adducts (Supporting Information Figure $\mathrm{S} 2 \mathrm{C}, \mathrm{R}^{2}=0.67$ ).
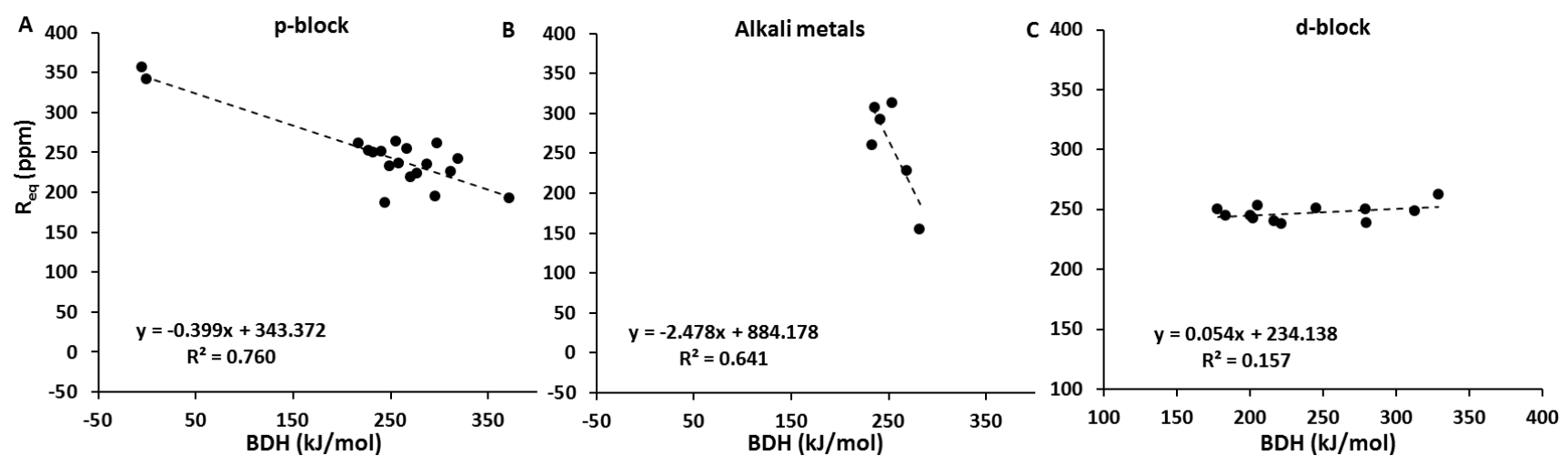

Figure 4. Correlation between equilibrium bond lengths (in pm) and bond dissociation enthalpy (in $\mathrm{kJ} / \mathrm{mol}$ ) for computed diatomic AuX molecules. A) $\mathrm{X}=$ p-block element. B) $\mathrm{X}=$ alkali metal. $\mathbf{C}) \mathrm{X}=$ d-block element. 
Diversity of Gold Bonds: Inversion of Dipole Moments. To understand these bonding patterns in more detail, the dipole moments of all AuX molecules were computed and collected in Figure 5 (numerical data in Supporting Information, Table S12). A positive (negative) value implies that the gold atom carries a positive (negative) partial charge within the molecule. Figure 5 shows clearly the point stressed above, that the bonding type is very diverse in these systems even though computational accuracy is fairly uniform (Figure 3): The typical standard deviation for dipole moments computed with different functionals is $0.2 \mathrm{D}$, thus the choice of method has relatively little effect on the polarity of the molecule, as is understandable as this is a density-derived (not energy-derived) property. The hybrid functionals and notably B2PLYP produce the most polar molecules, as shown previously ${ }^{39}$, due to the effect of HF exchange in polarizing electron clouds. Nevertheless the dipole moments are fairly similar for all methods considering the deviation relative to the magnitude of the dipole moments.

Some molecules are very covalent in their bonding behavior, such as $\mathrm{AuH}(1.1 \mathrm{D}$, using the PBE-D3 value), AuBe (0.2 D), AuSi (0.1 D), AuGe (0.7 D), AuSb (0.1 D), AuBi (0.8 D), and of course AuAu. The van der Waals complexes obviously have small dipole moments as well (0.4-0.9 D). The alkali metals and alkaline earth metals force highly ionic bonding with gold, forcing gold to keep one or even formally two additional electrons with dipole moments of $\sim 6$ and $7 \mathrm{D}$ for AuLi and AuNa and 9-10 D for AuK, AuRb, and AuCs (Table S12). In the other extreme, the halides force dipole moments that are also large (albeit somewhat smaller) but now inverted, with $\mathrm{AuF}$ (3.9 D), $\mathrm{AuCl}$ (3.4 D), $\mathrm{AuBr}$ (3.1 D), and AuI (2.5 D) having dipole moments of 2.5-3.9 D in a monotonic, decreasing order as the electronegativity decreases down through the periods. Thus, the computations reveal trends across the periodic table with main group 6 and 7 adducts showing substantially positively charged gold and main group 1 showing substantial negative charge, creating dipole moments as large as $10 \mathrm{D}$; these data testify to the diverse bonding features of gold, with full inversion from ionic via covalent to ionic in the opposite direction across the periodic table. 


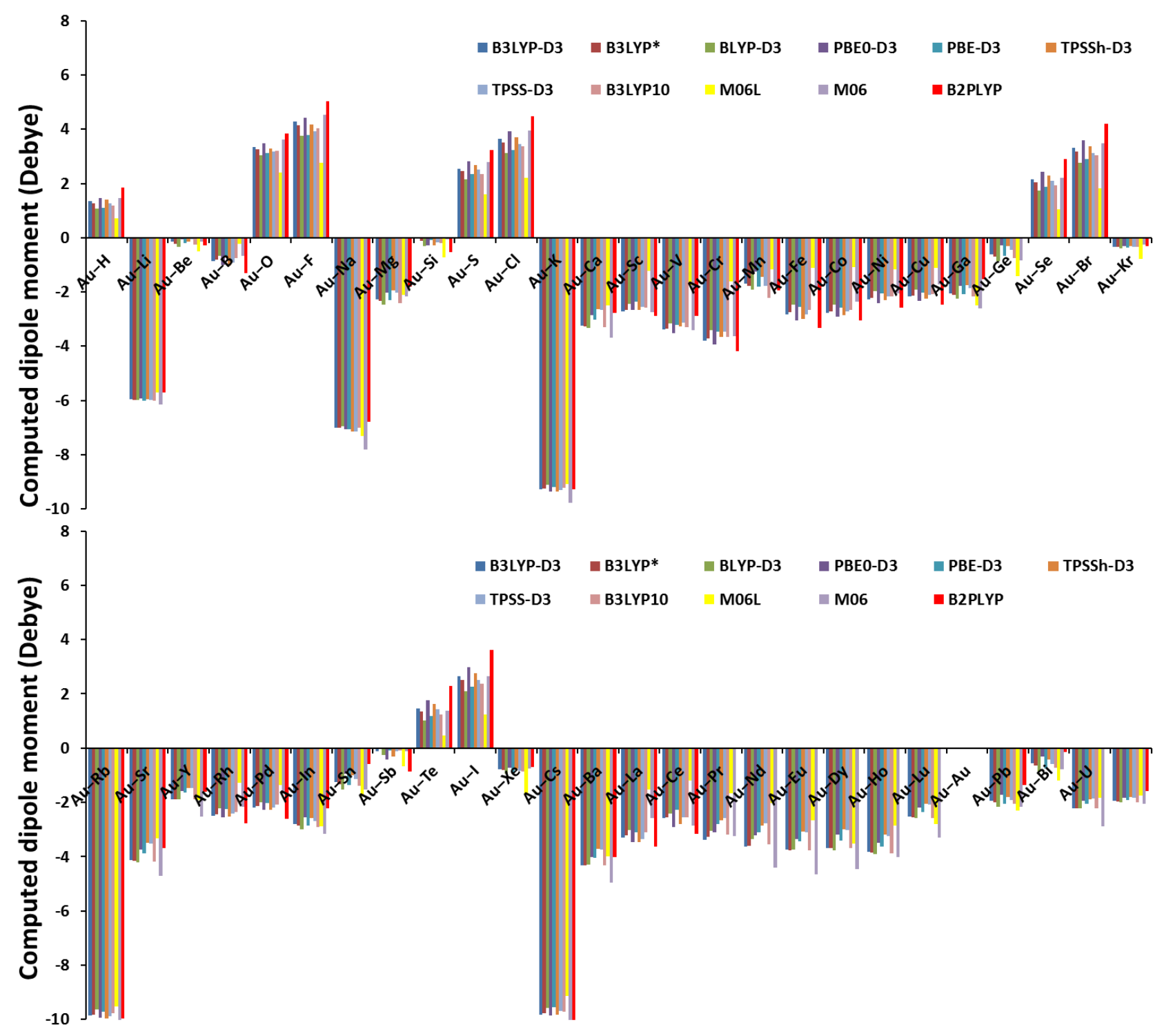

Figure 5. Computed dipole moments (in Debye) for diatomic AuX molecules. A positive value implies that $\mathrm{Au}$ is positively charged, and a negative value implies that Au is negatively charged.

Thiophilicity of Gold and Preference for Larger Chalcogenides. As a test of the trend accuracy of the methods in providing the correct relative features regardless of potential systematic errors, the important challenge of gold's extreme thiophilicity was studied ${ }^{4}$. It is well known that gold has a substantial preference for softer chalcogens such as sulfur and selenium, compared to oxygen. 
This is evident already from the mineral deposits ${ }^{54,55}$, is suspected based on the hard-soft Lewis acid base principle ${ }^{56,57,58}$, and is seen directly in the larger experimental BDH of AuS and AuSe (254 and $251 \mathrm{~kJ} / \mathrm{mol})$ vs. AuO $(223 \mathrm{~kJ} / \mathrm{mol})$. Yet, the value of AuTe $(237 \mathrm{~kJ} / \mathrm{mol})$ is in between these numbers despite being the softest of the chalcogens, suggesting that hardness does not explain this preference alone (Supporting Information, Table S1), as explained previously ${ }^{4}$

To test whether the density functionals are capable of reproducing this order, the differential $\mathrm{BDHs}$ relative to $\mathrm{AuO}$ were compared in Table 2. The experimental uncertainty in each separate BDH is $15-21 \mathrm{~kJ} / \mathrm{mol}$ but the experimental uncertainty in the differential BDHs is probably smaller due to systematic error components in the experimental protocol (the likelihood that the full error is $100 \%$ random is small). In all cases, the experimental data show that gold binds more strongly by $14-31$ $\mathrm{kJ} / \mathrm{mol}$ to the heavier chalcogens than to oxygen, consistent with the thiophilic nature of gold ${ }^{4}$. The experimental difference between $\mathrm{AuS}$ and $\mathrm{AuO}$ is $31 \mathrm{~kJ} / \mathrm{mol}$. It is encouraging that all methods can reproduce this difference with the correct sign (although M06L is close to failing), i.e. all methods predict that gold binds more strongly to sulfur than to oxygen, although the $25 \%$ and $27 \%$ hybrids PBE0 and M06 probably overestimate the difference, considering that the experimental error bars are upper bounds.

Considering the experimental uncertainties, Table 2 shows the semi-quantitative power of DFT applied to trend chemistry instead of absolute data. However, the different behavior even for trend chemistry is notable: Assuming small errors in the experimental data, TPSS gives a superior result even to PBE, apparently improving the trend chemistry, with some hybrid functionals also showing better trend behavior than their absolute numerical accuracy would imply (due to cancelation of systematic under-binding errors). Again $\operatorname{CCSD}(\mathrm{T})$ works well even at this basis set level in semi-quantitative estimates and is similar in numerical performance to the $10 \%$ hybrids TPSSh and B3LYP10 for the trend chemistry of Table 2 . 
Table 2. Computed Differences in Bond Dissociation Enthalpy of AuO vs. the Chalcogenides AuS, AuSe, and AuTe (kJ/mol), and Calculated Oxophilicity $\theta$.

\begin{tabular}{|c|c|c|c|c|c|c|c|c|c|c|c|c|c|c|}
\hline & $\begin{array}{l}\text { B3L } \\
\text { YP- } \\
\text { D3 }\end{array}$ & $\begin{array}{l}\text { B3L } \\
\text { YP* }\end{array}$ & $\begin{array}{l}\text { BLY } \\
\text { P-D3 }\end{array}$ & $\begin{array}{l}\text { PBE } \\
\text { 0-D3 }\end{array}$ & $\begin{array}{l}\text { PBE- } \\
\text { D3 }\end{array}$ & $\begin{array}{l}\text { TPSS } \\
\text { h-D3 }\end{array}$ & $\begin{array}{l}\text { TPSS } \\
-\mathrm{D3}\end{array}$ & $\begin{array}{l}\text { B3L } \\
\text { YP }\end{array}$ & $\begin{array}{l}\text { B3L } \\
\text { YP10 }\end{array}$ & $\begin{array}{l}\text { M06 } \\
\text { L }\end{array}$ & M06 & $\begin{array}{l}\text { B2P } \\
\text { LYP }\end{array}$ & $\begin{array}{l}\text { CCS } \\
\text { D(T) }\end{array}$ & Exp. $^{a}$ \\
\hline $\begin{array}{l}\mathrm{AuS}- \\
\mathrm{AuO}\end{array}$ & 37 & 30 & 8 & 54 & 19 & 44 & 31 & 37 & 23 & 3 & 57 & 47 & 46 & $31 \pm 26$ \\
\hline $\begin{array}{l}\mathrm{AuSe}- \\
\mathrm{AuO}\end{array}$ & 26 & 18 & -5 & 40 & 1 & 32 & 18 & 25 & 10 & -14 & 44 & 36 & 38 & $28 \pm 26$ \\
\hline $\begin{array}{l}\mathrm{AuTe}- \\
\mathrm{AuO}\end{array}$ & 17 & 8 & -17 & 30 & -12 & 22 & 7 & 16 & -1 & -17 & 48 & 28 & 27 & $14 \pm 26$ \\
\hline $\begin{array}{l}\text { Error }(\mathrm{S} \\
/ \mathrm{O})\end{array}$ & 6 & 0 & -22 & 23 & -12 & 14 & 0 & 6 & -7 & -27 & 27 & 16 & 16 & \\
\hline $\begin{array}{l}\text { Error(S } \\
\mathrm{e} / \mathrm{O})\end{array}$ & -2 & -10 & -33 & 12 & -27 & 4 & -10 & -3 & -18 & -42 & 16 & 8 & 10 & \\
\hline $\begin{array}{l}\text { Error( } \\
\text { Te/O) }\end{array}$ & 3 & -6 & -31 & 16 & -26 & 8 & -8 & 2 & -15 & -31 & 34 & 14 & 13 & \\
\hline$M A E$ & 4 & 6 & 29 & 17 & 22 & 9 & 6 & 4 & 14 & 34 & 25 & 13 & 13 & \\
\hline$\theta$ & 0.0 & 0.0 & 0.1 & 0.0 & 0.1 & 0.0 & 0.0 & 0.0 & 0.1 & 0.1 & -0.1 & 0.0 & 0.0 & $\begin{array}{l}0.04^{\text {ref. }} \\
4\end{array}$ \\
\hline
\end{tabular}

${ }^{\mathrm{a}}$ The estimated error was calculated as the square root of the sum of the squared errors, assuming random errors.

The last row of Table 2 shows the oxophilcity $\boldsymbol{\theta}$ calculated with each method based on the recently proposed formula ${ }^{4} \boldsymbol{\theta}=0.0035\left(\mathrm{BDH}_{\mathrm{AuO}}-\mathrm{BDH}_{\mathrm{AuS}}\right)+0.15$. This scales generally goes from 0-1 with 1 representing highly oxophilic systems and 0 representing extremely thiophilic systems. The value derived from the experimental numbers is $0.04 .^{4}$ As can be seen, all methods predict the same extremely low oxophilicity of gold $0.0 \pm 0.1$ within the stated error of the scale $(0.1)^{4}$, illustrating the power of theoretical chemistry for comparative chemistry where systematic errors cancel out. The experimental difference between AuSe/AuTe and AuO is clearly harder to compute accurately: BLYPD3 and M06L do not predict the correct order of bond strengths in either of these cases, and PBE-D3 underestimates the difference too. The hybrid functionals, in particular B3LYP-D3, and CCSD(T) and TPSS-D3 produce this difference most accurately. In summary, among the DFT methods, TPSS-D3, 
and some hybrid functionals, notably B3LYP-D3, produce the most accurate trend chemistry for gold's thiophilicity, making TPSS-D3 our remaining preferred overall choice at this point of the benchmark.

Bonding Trends in Gold Halides. Another relevant test data set is the relative bond strengths of gold bonds to the halides, where gold is mainly present in the $\mathrm{Au}^{+}$state. The corresponding analysis of these bonds is shown in Table 3. According to experimental thermochemical data, the AuF (294 $\mathrm{kJ} / \mathrm{mol})$ bond is the strongest, closely followed by that of $\mathrm{AuCl}(280 \mathrm{~kJ} / \mathrm{mol})$ and $\mathrm{AuI}(276 \mathrm{~kJ} / \mathrm{mol})$, with a major anomaly being the very low value for $\mathrm{AuBr}$ in between these $(213 \mathrm{~kJ} / \mathrm{mol}$, much smaller than the estimated uncertainty of $20 \mathrm{~kJ} / \mathrm{mol}$ ). An interesting question is how the methods handle this particular anomaly.

As seen from Table 3, the differential bonding of gold to the halides is qualitatively correct in almost all cases. For the difference in bond strength of $\mathrm{AuF}$ and $\mathrm{AuCl}$, the correct sign is produced by all methods except the two hybrids with most HF exchange, PBE0 and M06 (CCSD(T) is $1 \mathrm{~kJ} / \mathrm{mol}$ but within the uncertainty). All methods predict the correct order with substantial weaker binding of gold to $\mathrm{Br}$, but the numerical error is substantial, and all methods underestimate the difference between $\mathrm{AuBr}$ and AuF. Thus, this test set is indeed a challenging one for theoretical gold chemistry, and it would be interesting to see how other theoretical methods reproduce the anomaly of AuBr, assuming of course that the experimental data are not revised. For the large polarizable AuI, the loss of ionic bonding strength is compensated by stronger polarizability and covalency, such that the bond strengths of AuF and AuI become remarkably similar. The methods can reproduce this trend with the correct sign, although the numerical error is large due to weaknesses in the treatment of dispersion and differential relativistic effects of the heavy AuI vs. AuF. Overall, the MAEs for the test set are similar and of moderate value for all methods $(24-35 \mathrm{~kJ} / \mathrm{mol})$. In summary, all the studied DFT methods work well for semi-quantitative trends but the halide trend data constitute a difficult test case and shows that caution should be exercised when using DFT for trends down through the periods. 
Table 3. Computed Differences in Bond Dissociation Enthalpy of AuF vs. the Halides AuCl, AuBr, and AuI (kJ/mol).

\begin{tabular}{|l|l|l|l|l|l|l|l|l|l|l|l|l|l|l|}
\hline & $\begin{array}{l}\text { B3LY } \\
\text { P-D3 }\end{array}$ & $\begin{array}{l}\text { B3LY } \\
\text { P* }\end{array}$ & $\begin{array}{l}\text { BLYP- } \\
\text { D3 }\end{array}$ & $\begin{array}{l}\text { PBE0 } \\
\text { D3 }\end{array}$ & $\begin{array}{l}\text { PBE- } \\
\text { D3 }\end{array}$ & $\begin{array}{l}\text { TPSS } \\
\text { h-D3 }\end{array}$ & $\begin{array}{l}\text { TPSS- } \\
\text { D3 }\end{array}$ & $\begin{array}{l}\text { B3LY } \\
\text { P }\end{array}$ & $\begin{array}{l}\text { B3LY } \\
\text { P10 }\end{array}$ & M06L & M06 & $\begin{array}{l}\text { B2PL } \\
\text { YP }\end{array}$ & $\begin{array}{l}\text { CCSD } \\
\text { (T) }\end{array}$ & $\begin{array}{l}\text { Exp. } \\
\text { a }\end{array}$ \\
\hline $\begin{array}{l}\text { AuCl- } \\
\text { AuF }\end{array}$ & -11 & -17 & -33 & 10 & -18 & -2 & -13 & -12 & -22 & -16 & 5 & -7 & 1 & $-14 \pm 18$ \\
\hline $\begin{array}{l}\text { AuBr- } \\
\text { AuF }\end{array}$ & -28 & -34 & -52 & -7 & -38 & -17 & -29 & -28 & -40 & -46 & -30 & -22 & -12 & $-81 \pm 25$ \\
\hline $\begin{array}{l}\text { AuI-A } \\
\text { uF }\end{array}$ & -45 & -52 & -69 & -24 & -56 & -34 & -46 & -46 & -58 & -54 & -40 & -39 & -31 & $-18 \pm 30$ \\
\hline $\begin{array}{l}\text { Error( } \\
\text { Cl/F) }\end{array}$ & 3 & -2 & -19 & 24 & -4 & 12 & 1 & 3 & -8 & -2 & 20 & 7 & 15 & \\
\hline $\begin{array}{l}\text { Error( } \\
\text { Br/F) }\end{array}$ & 53 & 47 & 30 & 74 & 43 & 64 & 52 & 53 & 41 & 35 & 51 & 59 & 69 & \\
\hline $\begin{array}{l}\text { Error(I } \\
\text { F) }\end{array}$ & -27 & -34 & -51 & -6 & -38 & -16 & -28 & -28 & -40 & -36 & -22 & -21 & -13 & \\
\hline MAE & 28 & 28 & 33 & 35 & 28 & 31 & 27 & 28 & 30 & 24 & 31 & 29 & 32 & \\
\hline
\end{tabular}

${ }^{a}$ The estimated error was calculated as the square root of the sum of the squared errors, assuming random errors, and assuming that errors of $\mathrm{AuF} \sim \mathrm{AuCl}$, and $\mathrm{AuI} \sim \mathrm{AuBr}$.

Gold Trimers. Gold atoms form stable trimers that can be in various isomer forms, most notably a linear structure of $\mathrm{D}_{\infty \mathrm{h}}$ with $180^{\circ}$ and a triangular structure with $\mathrm{D}_{3 \mathrm{~h}}$ symmetry, and in between these, a bent structure with $\mathrm{C}_{2 \mathrm{v}}$ symmetry ${ }^{59}$. This makes the trimers a valuable test case for studying theoretical gold chemistry. It turns out that the $\mathrm{D}_{3 \mathrm{~h}}$ structure is not stable and converts into the $\mathrm{C}_{2 \mathrm{v}}$ isomer. This feature is obtained readily by DFT when starting from a triangular structure: As seen from the geometric data compiled in Table 4, the optimized $\mathrm{Au}-\mathrm{Au}-\mathrm{Au}$ angle of the bent trimer is $56^{\circ}$, in very good agreement with the angle of $55^{\circ}$ just reported by Barrow et al. ${ }^{59}$ Both TPSSh and PBE give similar structures to within $0.1^{\circ}$ and $1.2 \mathrm{pm}$, despite the differences in energies that they produce, confirming the picture from the AuX molecules that the choice of functional for geometry optimization is not very important. Interestingly, both methods predict a bent structure of $\mathrm{C}_{2 \mathrm{v}}$ symmetry which still has a significant interaction between the two terminal Au atoms, with an $\mathrm{Au}-\mathrm{Au}$ distance of $\sim 294 \mathrm{pm}$. 
Table 4. Computed Au-X Bond Lengths (in pm) and Au-X-Y angles for Gold-Molecule Interactions ( $\mathrm{X}=$ atom closest to gold).

\begin{tabular}{|c|c|c|c|c|}
\hline Molecule & $\begin{array}{l}\text { PBE-D3 } \\
\text { Au-X (ppm) }\end{array}$ & $\begin{array}{l}\text { PBE-D3 } \\
\angle A u-X-Y\left(^{\circ}\right)\end{array}$ & $\begin{array}{l}\text { TPSSh-D3 } \\
\text { Au-X (ppm) }\end{array}$ & $\begin{array}{l}\text { TPSSh-D3 } \\
\angle A u-X-Y\left(^{\circ}\right)\end{array}$ \\
\hline Linear $\mathrm{Au}_{3}$ & $258.8,258.7$ & 180.0 & $258.0,258.0$ & 180.0 \\
\hline Bent $\mathrm{Au}_{3}$ & $262.2,262.5$ & 56.0 & $261.0,261.7$ & 56.1 \\
\hline $\mathrm{AuCO}^{+}$ & 190.5 & 180.0 & 192.7 & 180.0 \\
\hline $\mathrm{Au}-\mathrm{NH}_{3}$ & 234.4 & 109.9 & 235.4 & 110.2 \\
\hline $\mathrm{Au}-\mathrm{CH}_{3}$ & 202.0 & 107.1 & 202.4 & 107.3 \\
\hline $\mathrm{Au}-\mathrm{C}_{6} \mathrm{H}_{6}$ & 230.7 & $91.3-98.9$ & 239.4 & $91.4-96.4$ \\
\hline
\end{tabular}

The computations consistently show that both trimers have a doublet ground state, with the quartet substantially higher in energy $(262 \mathrm{~kJ} / \mathrm{mol}$ and $146 \mathrm{~kJ} / \mathrm{mol}$ in the linear and triangular structures, respectively), which is expected due to the tight coupling of the 6 s electrons in the threecenter $\sigma$-bond of the trimers.

To assess the performance of the different functionals for the thermochemistry of the gold trimer, the total bond enthalpy of the trimers (including two $\mathrm{Au}-\mathrm{Au}$ bonds in these structures) was computed as the energy of the trimers minus the energy of three gold atoms, correcting for dispersion and thermal and vibrational contributions as for the AuX systems. Table 5 lists these total BDH values for the linear and bent $\mathrm{Au}_{3}$ isomers. Again, when it comes to the absolute thermochemistry computed with the different functionals, the differences are substantial, confirming the picture seen for the AuX 
molecules, and reflecting the different treatments of the atomic vs. molecular states. The hybrids predict substantially weaker $\mathrm{Au}-\mathrm{Au}$ bonds, e.g. the difference is $64 \mathrm{~kJ} / \mathrm{mol}$ between B3LYP and PBED3. The strongest adducts are obtained with M06L, followed by PBE and TPSS. However, the same systematic error is present in both trimers, and the atomic states where most of the static correlation resides cancel, so if one is interested in the enthalpy of reaction for converting one isomer into the other (the relative stability of the two isomers) all studied functionals provide results in very good agreement, favoring the bent isomer over the linear isomer by 3-15 kJ/mol. The predicted weak favoring of the bent isomer may perhaps be confirmed in future experiments, as it seems robust because of systematic error cancellation. The prediction that bent $\mathrm{Au}_{3}$ is more stable than linear $\mathrm{Au}_{3}$ is partly due to the fact, not included in Table 4, that also the larger vibrational entropy favors the bent structure (by a computed differential entropy of $90 \mathrm{~J} / \mathrm{molK}$, based on the harmonic frequency state functions of the two states).

Table 5. Computed Total Bond Dissociation Enthalpies (Including Thermal and Vibrational Corrections) of Linear and Triangular Gold Trimers and the Enthalpy Difference between the Two Trimers (All values are in $\mathrm{kJ} / \mathrm{mol}$ ).

\begin{tabular}{|l|l|l|l|l|l|l|l|}
\hline & $\begin{array}{l}\text { B3LYP- } \\
\text { D3 }\end{array}$ & B3LYP* & $\begin{array}{l}\text { BLYP- } \\
\text { D3 }\end{array}$ & $\begin{array}{l}\text { PBE0- } \\
\text { D3 }\end{array}$ & PBE-D3 & $\begin{array}{l}\text { TPSSh- } \\
\text { D3 }\end{array}$ & $\begin{array}{l}\text { TPSS- } \\
\text { D3 }\end{array}$ \\
\hline Linear & 259 & 269 & 287 & 271 & 317 & 295 & 314 \\
\hline Bent & 249 & 257 & 272 & 268 & 309 & 292 & 310 \\
\hline$\Delta \mathrm{H}^{\mathbf{o}}{ }_{\mathrm{r}}$ & -10 & -12 & -15 & -3 & -8 & -3 & -5 \\
\hline & B3LYP & B3LYP10 & M06L & M06 & B2PLYP & CCSD(T) & \\
\hline Linear & 253 & 286 & 344 & 283 & 267 & 263 & \\
\hline Bent & 240 & 274 & 318 & 279 & 262 & 263 & \\
\hline$\Delta \mathrm{H}^{\mathrm{o}}{ }_{\mathrm{r}}$ & -13 & -12 & -26 & -4 & -5 & 1 & \\
\hline
\end{tabular}


Table 6. Computed Bond Dissociation Enthalpies (in $\mathrm{kJ} / \mathrm{mol}$ ) for Other Gold-Molecule Interactions.

\begin{tabular}{|c|c|c|c|c|c|c|c|c|}
\hline Molecule & $\begin{array}{l}\text { B3LYP- } \\
\text { D3 }\end{array}$ & B3LYP* & $\begin{array}{l}\text { BLYP- } \\
\text { D3 }\end{array}$ & $\begin{array}{l}\text { PBE0- } \\
\text { D3 }\end{array}$ & PBE-D3 & $\begin{array}{l}\text { TPSSh- } \\
\text { D3 }\end{array}$ & $\begin{array}{l}\text { TPSS- } \\
\text { D3 }\end{array}$ & Exp \\
\hline $\mathrm{AuCO}^{+}$ & 188 & 198 & 215 & 201 & 242 & 209 & 225 & $199 \pm 11$ \\
\hline $\mathrm{Au}-\mathrm{NH}_{3}$ & 15 & 14 & 21 & 19 & 31 & 23 & 28 & $76 \pm 6$ \\
\hline $\mathrm{Au}-\mathrm{CH}_{3}$ & 218 & 227 & 235 & 229 & 258 & 232 & 242 & $>191.6$ \\
\hline $\mathrm{Au}-\mathrm{C}_{6} \mathrm{H}_{6}$ & 7 & 0 & 18 & 17 & 32 & 23 & 29 & 8.4 \\
\hline $\mathrm{AuXe}^{+}$ & 111 & 119 & 128 & 117 & 142 & 120 & 129 & $130 \pm 13$ \\
\hline $\mathrm{AuH}^{+}$ & 190 & 200 & 211 & 179 & 211 & 200 & 212 & $209 \pm 11$ \\
\hline $\mathrm{AuNH}_{3}{ }^{+}$ & 249 & 257 & 267 & 254 & 282 & 260 & 271 & $297 \pm 29$ \\
\hline$M S E$ & -19 & -14 & -2 & -14 & 12 & -6 & 4 & \\
\hline$M A E$ & 26 & 24 & 22 & 27 & 30 & 25 & 25 & \\
\hline Molecule & $\overline{B 3 L Y P}$ & $\overline{\text { B3LYP10 }}$ & M06L & M06 & B2PLYP & $\overline{\operatorname{CCSD}(\mathrm{T})}$ & & Exp \\
\hline $\mathrm{AuCO}^{+}$ & 184 & 212 & 228 & 167 & 189 & 187 & & $199 \pm 11$ \\
\hline $\mathrm{Au}-\mathrm{NH}_{3}$ & 9 & 20 & 43 & 13 & 27 & 36 & & $76 \pm 6$ \\
\hline $\mathrm{Au}-\mathrm{CH}_{3}$ & 215 & 238 & 252 & 222 & 233 & 246 & & $>191.6$ \\
\hline $\mathrm{Au}-\mathrm{C}_{6} \mathrm{H}_{6}$ & -8 & 7 & 41 & 7 & 8 & 20 & & 8.4 \\
\hline $\mathrm{AuXe}^{+}$ & 110 & 128 & 148 & 112 & 105 & 95 & & $130 \pm 13$ \\
\hline $\mathrm{AuH}^{+}$ & 190 & 209 & 219 & 143 & 176 & 167 & & $209 \pm 11$ \\
\hline $\mathrm{AuNH}_{3}{ }^{+}$ & 246 & 268 & 288 & 231 & 247 & 263 & & $297 \pm 29$ \\
\hline$M S E$ & -24 & -4 & 15 & -31 & -18 & -14 & & \\
\hline$M A E$ & 30 & 21 & 28 & 39 & 30 & 33 & & \\
\hline
\end{tabular}


Other Gold-Containing Molecules and Cations. To further illustrate the performance of the functionals, the BDHs of some additional gold-containing molecules with known experimental BDHs were computed: $\mathrm{AuCO}+, \mathrm{Au}-\mathrm{NH}_{3}$ and its cation $\mathrm{Au}-\mathrm{NH}_{3}{ }^{+}, \mathrm{Au}-\mathrm{CH}_{3}$, the gold atom associated with benzene, $\mathrm{Au}-\mathrm{C}_{6} \mathrm{H}_{6}$, as well as the diatomic cations $\mathrm{AuH}^{+}$and $\mathrm{AuXe}^{+}$. These systems represent a broad range of interactions, including a coordinate bonds in $\mathrm{Au}-\mathrm{NH}_{3}$ and $\mathrm{Au}-\mathrm{NH}_{3}{ }^{+}$, covalent organometallic bonds in $\mathrm{AuCO}^{+}$and $\mathrm{Au}-\mathrm{CH}_{3}$, the $\mathrm{AuH}^{+}$system which is notable by itself as a common test case of gold chemistry ${ }^{19}$, a noble gas adduct with the $\mathrm{Au}^{+}$ion, and very weak interactions between the polarizable gold atom and the $\pi$-system of benzene, which is very dispersion-dependent. These results are collected in Table 6. As can be seen, the computed BDHs follow the very same trend of bond strength as seen for the other systems, suggesting strongly that the bond weakening effect of HF exchange is general for all these types of chemical bonds to gold. As for the diatomic neutral molecules, it is encouraging that all methods can model these bonds with good numerical accuracy but the data set is too small to make up conclusions on the performance of the functionals; they tend to perform similar overall for this data set, with MAEs of 22-30 kJ/mol, except M06 which has an MAE of $39 \mathrm{~kJ} / \mathrm{mol}$; again, the performance of $\operatorname{CCSD}(\mathrm{T})$ resembles that of average DFT methods when applying a def2-TZVP basis set, but accuracy can surely be increased for this method separately by increasing the basis $\operatorname{set}^{29}$.

The $\mathrm{Au}-\mathrm{C}_{6} \mathrm{H}_{6}$ adduct is special in this benchmark as it probes very weak interactions with gold. It is notable that DFT without dispersion is incapable of predicting any binding between a gold atom and a benzene ring: $\operatorname{CCSD}(\mathrm{T})$ models this interaction well, as do some of the functionals with dispersion included. The two methods that do not account for dispersion, B3LYP* with $15 \%$ HF exchange and B3LYP without D3 correction, predict zero or negative $\mathrm{BDH}$ for $\mathrm{Au}-\mathrm{C}_{6} \mathrm{H}_{6}$. This structure is also notable by being asymmetric, and not a classical $\mathrm{D}_{6 \mathrm{~h}}$ interaction as might be expected, because of the propensity of gold to form a $\sigma$ interaction with one carbon, an interaction that however still has a 
large dispersion contribution. This shows that dispersion is critical for this particular system, making it a good test case for this type of weak-bonding theoretical gold chemistry.

Concluding Remarks. This paper has reported a systematic benchmark of commonly used density functionals with effective core potentials applied to the modelling of diverse chemical bonds to gold. As far as the author is aware, it is the first of its kind, although hundreds of papers report studies using this very approximate methodology. Thus, the initial question was very skeptical: How accurate are these approaches really, considering the complexity, dispersion and relativistic effects of gold? The main purpose was to identify a DFT method, if any, that can provide fast accurate estimates of chemical bonds to gold.

While the results clearly show that density functionals give highly variable results for chemical bonds to gold, these variations can be systematically understood from the contribution of HF exchange and the LYP functional, and zero point energies in lowering bond strengths, whereas dispersion strengthens bonds to gold. Specific examples are given that serve as useful test cases for theoretical gold chemistry, and predictions on the nature of the most stable gold trimer and the ground states of many AuX molecules are presented.

A surprisingly positive conclusion from this work is that research using PBE or TPSS functionals with dispersion corrections perform uniformly well except for a few pathological cases, with mean absolute errors of $23 \mathrm{~kJ} / \mathrm{mol}$, quite respectable in comparison to most other computational chemistry considering the implicit approximations in the functionals and the use of effective core potentials to model the relativistic bond contraction. Altogether, including trend chemistry, absolute and signed errors, TPSS performs most accurately, mirroring previous findings for the specific case of gold carbon adducts ${ }^{28}$. Thus, rather than enforcing extensive spin-orbit coupling schemes that would prevent standard applications towards larger systems in nanochemistry and catalysis, the TPSS + 
effective core approach, if electronic configurations are carefully optimized, uniform basis sets and effective core potentials are used, and zero-point vibrational corrections and dispersion is accounted for, works quite well, which is highly encouraging.

Supporting Information Available. The Supporting Information file includes the experimental bond dissociation enthalpies in Table S1 and Table S2 with error bars where available; optimized equilibrium bond lengths in pm (Table S3); computed electronic energies of molecules with lowest spin state marked in bold (Table S4) and for ground states with all studied methods (Table S5); electronic energies of atoms (Table S6); Thermal and vibrational corrections to energies (Table S7); computed bond dissociation enthalpies of diatomic (Table S8) and polyatomic molecules (Table S9); signed errors for all systems and methods (Table S10); absolute errors for all systems and methods (Table S11): and computed dipole moments with all methods (Table S12); computed coupled-cluster D1 diagnostics for AuX systems (Figure S1); trends in computed BDHs vs. Au-X bond lengths (Figure $\mathrm{S} 2)$. This material is available free of charge via the Internet at http:/pubs.acs.org.

Acknowledgements. The Danish Center for Scientific Computing / Aarhus Supercomputer Center is gratefully acknowledged for providing the computational resources for the project. 


\section{References.}

(1) Pyykkö, P. Theoretical Chemistry of Gold. Angew. Chemie Int. Ed. 2004, 43, 4412-4456.

(2) Pyykkö, P. Theoretical Chemistry of Gold. III. Chem. Soc. Rev. 2008, 37, 1967-1997.

(3) Gorin, D. J.; Toste, F. D. Relativistic Effects in Homogeneous Gold Catalysis. Nature 2007, 446, $395-403$.

(4) Kepp, K. P. A Quantitative Scale of Oxophilicity and Thiophilicity. Inorg. Chem. 2016, 55, 9461-9470.

(5) Wang, B.; Yin, S.; Wang, G.; Buldum, A.; Zhao, J. Novel Structures and Properties of Gold Nanowires. Phys. Rev. Lett. 2001, 86, 2046.

(6) Häkkinen, H. The Gold-Sulfur Interface at the Nanoscale. Nat. Chem. 2012, 4, 443-455.

(7) Mannini, M.; Pineider, F.; Sainctavit, P.; Danieli, C.; Otero, E.; Sciancalepore, C.; Talarico, A. M.; Arrio, M.-A.; Cornia, A.; Gatteschi, D. Magnetic Memory of a Single-Molecule Quantum Magnet Wired to a Gold Surface. Nat. Mater. 2009, 8, 194-197.

(8) Häkkinen, H. Atomic and Electronic Structure of Gold Clusters: Understanding Flakes, Cages and Superatoms from Simple Concepts. Chem. Soc. Rev. 2008, 37, 1847-1859.

(9) Zhang, J.; Sasaki, K.; Sutter, E.; Adzic, R. R. Stabilization of Platinum Oxygen-Reduction Electrocatalysts Using Gold Clusters. Science 2007, 315, 220-222.

(10) Turner, M.; Golovko, V. B.; Vaughan, O. P. H.; Abdulkin, P.; Berenguer-Murcia, A.; Tikhov, M. S.; Johnson, B. F. G.; Lambert, R. M. Selective Oxidation with Dioxygen by Gold 
Nanoparticle Catalysts Derived from 55-Atom Clusters. Nature 2008, 454, 981-983.

(11) Oliver-Meseguer, J.; Cabrero-Antonino, J. R.; Domínguez, I.; Leyva-Pérez, A.; Corma, A. Small Gold Clusters Formed in Solution Give Reaction Turnover Numbers of 107 at Room

Temperature. Science 2012, 338, 1452-1455.

(12) Schmidbaur, H.; Schier, A. Aurophilic Interactions as a Subject of Current Research: An upDate. Chem. Soc. Rev. 2012, 41, 370-412.

(13) Kepp, K. P. Consistent Descriptions of Metal-ligand Bonds and Spin-Crossover in Inorganic Chemistry. Coord. Chem. Rev. 2013, 257, 196-209.

(14) Becke, A. D. Perspective: Fifty Years of Density-Functional Theory in Chemical Physics. $J$. Chem. Phys. 2014, 140, 18A301.

(15) Yang, W.; Hashmi, A. S. K. Mechanistic Insights into the Gold Chemistry of Allenes. Chem. Soc. Rev. 2014, 43, 2941-2955.

(16) Fang, H.-C.; Li, Z. H.; Fan, K.-N. CO Oxidation Catalyzed by a Single Gold Atom: Benchmark Calculations and the Performance of DFT Methods. Phys. Chem. Chem. Phys. 2011, 13, 1335813369.

(17) Ciancaleoni, G.; Rampino, S.; Zuccaccia, D.; Tarantelli, F.; Belanzoni, P.; Belpassi, L. An Ab Initio Benchmark and DFT Validation Study on Gold (I)-Catalyzed Hydroamination of Alkynes. J. Chem. Theory Comput. 2014, 10, 1021-1034.

(18) Goel, S.; Velizhanin, K. A.; Piryatinski, A.; Tretiak, S.; Ivanov, S. A. DFT Study of Ligand Binding to Small Gold Clusters. J. Phys. Chem. Lett. 2010, 1, 927-931.

(19) Pyykkö, P. Theoretical Chemistry of Gold. II. Inorganica Chim. Acta 2005, 358, 4113-4130. 
(20) Becke, A. D. Density-functional Thermochemistry. III. The Role of Exact Exchange. J. Chem. Phys. 1993, 98, 5648-5652.

(21) Lee, C.; Yang, W.; Parr, R. G. Development of the Colle-Salvetti Correlation-Energy Formula into a Functional of the Electron Density. Phys. Rev. B 1988, 37, 785.

(22) Colle, R.; Salvetti, O. Approximate Calculation of the Correlation Energy for the Closed Shells. Theor. Chim. Acta 1975, 37, 329-334.

(23) Wu, Z. J.; Kawazoe, Y. Electron Affinities and Ionization Potentials of 4d and 5d Transition Metal Atoms by CCSD (T), MP2 and Density Functional Theory. Chem. Phys. Lett. 2006, 423, 81-86.

(24) Perdew, J. P.; Burke, K.; Ernzerhof, M. Generalized Gradient Approximation Made Simple. Phys. Rev. Lett. 1996, 77, 3865.

(25) Grönbeck, H.; Curioni, A.; Andreoni, W. Thiols and Disulfides on the Au(111) Surface: The Headgroup-Gold Interaction. J. Am. Chem. Soc. 2000, 122, 3839-3842.

(26) Iori, F.; Corni, S.; Di Felice, R. Unraveling the Interaction between Histidine Side Chain and the Au(111) Surface: A DFT Study. J. Phys. Chem. C 2008, 112, 13540-13545.

(27) Pašteka, L. F.; Rajský, T.; Urban, M. Toward Understanding the Bonding Character in Complexes of Coinage Metals with Lone-Pair Ligands. CCSD(T) and DFT Computations. $J$. Phys. Chem. A 2013, 117, 4472-4485.

(28) Nava, P.; Hagebaum-Reignier, D.; Humbel, S. Bonding of Gold with Unsaturated Species. ChemPhysChem 2012, 13, 2090-2096.

(29) Ciccioli, A.; Gigli, G.; Lauricella, M. Experimental and Computational Investigation of the 
Group 11-group 2 Diatomic Molecules: First Determination of the AuSr and AuBa Bond Energies and Thermodynamic Stability of the Copper-and Silver-Alkaline Earth Species. J. Chem. Phys. 2012, 136, 184306.

(30) Jensen, K. P. Metal-Ligand Bonds of Second and Third Row D- Block Metals Characterized by Density Functional Theory. 2009, 1-5.

(31) Haynes, W. M. CRC Handbook of Chemistry and Physics; CRC press, 2014.

(32) Gingerich, K. A.; Finkbeiner, H. C. Experimental and Predicted Bond Energies of Gaseous Rare-Earth Aurides. J. Chem. Soc. D 1969, 16, 901-902.

(33) Ahlrichs, R.; Bär, M.; Häser, M.; Horn, H.; Kölmel, C. Electronic Structure Calculations on Workstation Computers: The Program System Turbomole. Chem. Phys. Lett. 1989, 162, 165169.

(34) Eichkorn, K.; Treutler, O.; Öhm, H.; Häser, M.; Ahlrichs, R. Auxiliary Basis Sets to Approximate Coulomb Potentials. Chem. Phys. Lett. 1995, 240, 283-290.

(35) Grimme, S.; Antony, J.; Ehrlich, S.; Krieg, H. A Consistent and Accurate Ab Initio Parametrization of Density Functional Dispersion Correction (DFT-D) for the 94 Elements HPu. J. Chem. Phys. 2010, 132, 154104.

(36) Tao, J.; Perdew, J. P.; Staroverov, V. N.; Scuseria, G. E. Climbing the Density Functional Ladder: Nonempirical Meta Generalized Gradient Approximation Designed for Molecules and Solids. Phys. Rev. Lett. 2003, 91, 146401.

(37) Perdew, J. P.; Tao, J.; Staroverov, V. N.; Scuseria, G. E. Meta-Generalized Gradient Approximation: Explanation of a Realistic Nonempirical Density Functional. J. Chem. Phys. 
2004, 120, 6898-6911.

(38) Weigend, F.; Ahlrichs, R. Balanced Basis Sets of Split Valence, Triple Zeta Valence and Quadruple Zeta Valence Quality for H to Rn: Design and Assessment of Accuracy. Phys. Chem. Chem. Phys. 2005, 7, 3297-3305.

(39) Jensen, K. P.; Roos, B. O.; Ryde, U. Performance of Density Functionals for First Row Transition Metal Systems. J. Chem. Phys. 2007, 126, 14103.

(40) Adamo, C.; Barone, V. Toward Reliable Density Functional Methods without Adjustable Parameters: The PBE0 Model. J. Chem. Phys. 1999, 110, 6158-6170.

(41) Stephens, P. J.; Devlin, F. J.; Chabalowski, Cf.; Frisch, M. J. Ab Initio Calculation of Vibrational Absorption and Circular Dichroism Spectra Using Density Functional Force Fields. J. Phys. Chem. 1994, 98, 11623-11627.

(42) Becke, A. D. Density-Functional Exchange-Energy Approximation with Correct Asymptotic Behavior. Phys. Rev. A 1988, 38, 3098-3100.

(43) Reiher, M.; Salomon, O.; Hess, B. A. Reparameterization of Hybrid Functionals Based on Energy Differences of States of Different Multiplicity. Theor. Chem. Acc. 2001, 107, 48-55.

(44) Salomon, O.; Reiher, M.; Hess, B. A. Assertion and Validation of the Performance of the

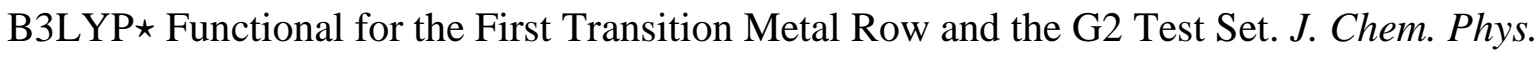
2002, 117, 4729-4737.

(45) Zhao, Y.; Truhlar, D. G. The M06 Suite of Density Functionals for Main Group Thermochemistry, Thermochemical Kinetics, Noncovalent Interactions, Excited States, and Transition Elements: Two New Functionals and Systematic Testing of Four M06-Class 
Functionals and 12 Other Function. Theor. Chem. Acc. 2008, 120, 215-241.

(46) Zhao, Y.; Truhlar, D. G. A New Local Density Functional for Main-Group Thermochemistry, Transition Metal Bonding, Thermochemical Kinetics, and Noncovalent Interactions. J. Chem. Phys. 2006, 125, 194101.

(47) Grimme, S. Semiempirical Hybrid Density Functional with Perturbative Second-Order Correlation. J. Chem. Phys. 2006, 124, 34108.

(48) Weigend, F.; Häser, M. RI-MP2: First Derivatives and Global Consistency. Theor. Chem. Acc. 1997, 97, 331-340.

(49) Hättig, C.; Weigend, F. CC2 Excitation Energy Calculations on Large Molecules Using the Resolution of the Identity Approximation. J. Chem. Phys. 2000, 113, 5154-5161.

(50) Pyykkö, P. Noblesse Oblige. Science 2000, 290, 64-65.

(51) Medvedev, M. G.; Bushmarinov, I. S.; Sun, J.; Perdew, J. P.; Lyssenko, K. A. Density Functional Theory Is Straying from the Path toward the Exact Functional. Science 2017, 355 (6320), 49-52.

(52) Jensen, K.; Ryde, U. Theoretical Prediction of the Co-C Bond Strength in Cobalamins. J. Phys. Chem. A 2003, 155, 7539-7545.

(53) Liu, W.; Dolg, M. Benchmark Calculations for Lanthanide Atoms: Calibration of Ab Initio and Density-Functional Methods. Phys. Rev. A 1998, 57, 1721.

(54) Schulze, D. G.; Dixon, J. B. An Introduction to Soil Mineralogy. Soil Mineral. with Environ. Appl. 2002, 1-35.

(55) Misra, K. Understanding Mineral Deposits; Springer Science \& Business Media, 2012. 
(56) Schwarzenbach, G. The General, Selective, and Specific Formation of Complexes by Metallic Cations. In; Radiochemistry, H. J. E. and A. G. S. B. T.-A. in I. C. and, Ed.; Academic Press, 1961; Vol. Volume 3, pp 257-285.

(57) Ahrland, S.; Chatt, J.; Davies, N. R. The Relative Affinities of Ligand Atoms for Acceptor Molecules and Ions. Q. Rev. Chem. Soc. 1958, 12, 265-276.

(58) Pearson, R. G. Hard and Soft Acids and Bases. J. Am. Chem. Soc. 1963, 85, 3533-3539.

(59) Barrow, S. J.; Collins, S. M.; Rossouw, D.; Funston, A. M.; Botton, G. A.; Midgley, P. A.; Mulvaney, P. Electron Energy Loss Spectroscopy Investigation into Symmetry in Gold Trimer and Tetramer Plasmonic Nanoparticle Structures. ACS Nano 2016, 10, 8552-8563. 
TOC Graphic

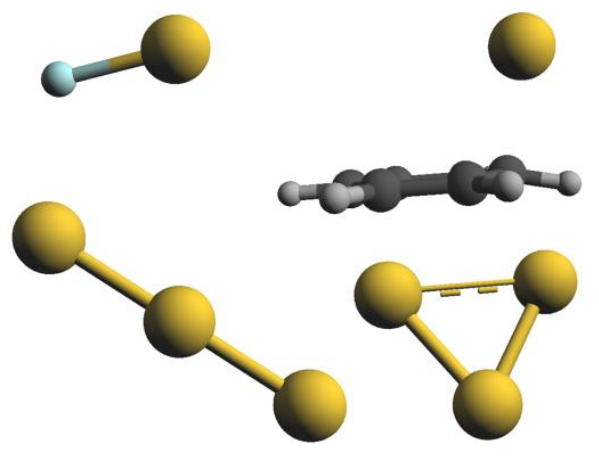

